\title{
Production of excitons in grazing collisions of protons with LiF surfaces: An onion model
}

\author{
J. E. Miraglia and M. S. Gravielle \\ Instituto de Astronomía y Física del Espacio, Consejo Nacional de Investigaciones Científicas y Técnicas and \\ Departamento de Física, Facultad de Ciencias Exactas y Naturales, Universidad de Buenos Aires, Casilla de Correo 67, \\ Sucursal 28, (C1428EGA) Buenos Aires, Argentina \\ (Received 8 July 2011; published 2 December 2011)
}

\begin{abstract}
In this work we evaluate the production of excitons of a lithium fluoride crystal induced by proton impact in the intermediate and high energy regime (from $100 \mathrm{keV}$ to $1 \mathrm{MeV}$ ). A simple model is proposed to account for the influence of the Coulomb grid of the target by dressing crystal ions to transform them in what we call onions. The excited states of these onions can be interpreted as excitons. Within this model, total cross section and stopping power are calculated by using the first Born and the continuum distorted-wave (CDW) eikonal initial-state (EIS) approximations. We found that between 7 and 30 excitons per incident proton are produced in grazing collisions with LiF surfaces, becoming a relevant mechanism of inelastic transitions.
\end{abstract}

DOI: 10.1103/PhysRevA.84.062901

PACS number(s): 34.50.Bw, 71.35.Aa, 79.20.Rf

\section{INTRODUCTION}

There is strong theoretical and experimental evidence that excitons play an important role in the charge transfer to neutral hydrogen atoms by grazing scattering from LiF surfaces at low impact velocities. In this process it has been proven that excitons are first excited as a consequence of the collision and then, from this intermediate state, the electron is captured by the projectile to form $\mathrm{H}^{-}$[1-5]. There is also a large number of experiments on radiation absorption where it is possible to excite excitons and, what is more interesting, the energy associated with this electronic excitation can travel in the periodic crystal carrying no electric charge [6]. In semiconductors excitons are depicted as an electron and a positive hole rotating around each other, much like a positron [7]. In the present article we deal with insulators and the focus is devoted to evaluate the formation probability of excitons by proton impact. After the collision, excitons are known to migrate (electron and hole) through the crystal, relaxing to give rise to photon emission (luminescence), defect creation, or nonradiative transitions (conversion of the electronic excitation energy into lattice vibrations) [6]. We do not pay attention to any of these post-collisional processes here.

To illustrate the focus of this work, let us first consider the stopping power of protons penetrating the LiF bulk at intermediate and high energies where experiments are available [8,9] [see Fig. 1(a)]. A basic theoretical approximation to interpret these experiments is to consider the target as a grid of independent ions (GII), composed by $\mathrm{F}^{-}$and $\mathrm{Li}^{+}$ions forming a NaCl-type structure. By independent ions we mean to isolated cations and anions whose electron wave functions are described, for example, by Clementi-Roetti Slater orbitals [10]. Within this model, the three principal mechanisms of projectile energy loss are $\mathrm{F}^{-}$and $\mathrm{Li}^{+}$ionizations [denoted in Fig. 1 as $\mathrm{F}^{-}(1+2 \rightarrow \varepsilon)$ and $\mathrm{Li}^{+}(1 \rightarrow \varepsilon)$, respectively], and $\mathrm{Li}^{+}$excitation $\left[\mathrm{Li}^{+}(1 \rightarrow 2+3)\right]$. The notation $1+2$ indicates that initial states belong to the shells $n=1$ and 2, while $2+3(\varepsilon)$ indicates transitions to the corresponding final excited shells (continuum states). In fact, the contribution of ionization from the $\mathrm{F}^{-}(1 s)$ state as well as of the electron capture process [11] are both negligible in the considered energy range. We also neglect the nuclear stopping (i.e., elastic collisions with the whole target ion) and excitation of lattice vibrations which contribute only at low projectile velocities [12]. The important point is that within the framework of the GII model there is no possible $\mathrm{F}^{-}$excitation because there is no excited state for such an isolated negative ion.

In Fig. 1(a) we show partial and total stopping cross sections corresponding to the above mentioned processes, which were derived with the GII model by using the first Born and continuum distorted-wave (CDW) eikonal initial-state (EIS) approximations. Calculations were performed rigorously by solving the radial wave functions for the bound and continuum states [13]. Both theories, Born and CDWEIS, seem to reproduce very well the data in almost the whole velocity range, starting to depart each other at low energies only. For impact energies lower than $100 \mathrm{keV}$ these theories display the usual behavior: Born and CDWEIS results overestimate and underestimate, respectively, the experiments, reflecting the range of validity of the approximations. In Fig. 1(b) we also show theoretical calculations of excitation and ionization cross sections as a function of the projectile velocity. The total inelastic cross section, obtained by adding the contributions of the different mechanisms, gives the inverse of the mean free path when multiplied by the density of ionic targets. Within the GII model, the main process is $\mathrm{F}^{-}$ionization, and consequently, the inelastic mean free path is completely governed by electron emission to the continuum.

In previous articles the GII model has successfully been used to treat a series of processes involving LiF crystals, such as electron production [14,15], stopping power [16], etc. We have even extended the GII model to deal with interference effects for He atoms colliding with a LiF surface along low-indexed crystallographic directions [17]. However, although the GII model predicts reasonably well different magnitudes [as shown in Fig. 1(a)], it is physically incomplete to describe NaCl-type crystals since it neglects the Coulomb interactions of the crystal lattice. There are two main effects that are not taken into account in the GII model. First, as the unperturbed crystal is neutral, after ionization the emitted electron should see its own hole at large distances; that is, regardless of its initial state, it should feel an attractive unitary Coulomb potential far from its parent target [18]. And second, 


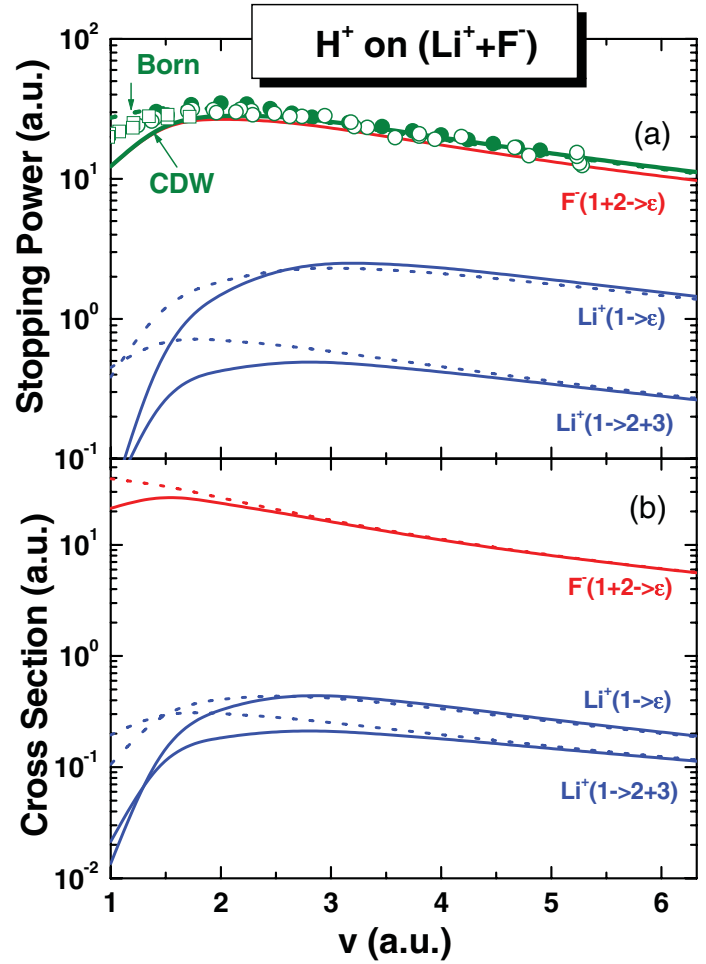

FIG. 1. (Color online) (a) Stopping power cross sections for protons moving in a $\mathrm{LiF}$ crystal as a function of the proton velocity. Full circles, experiments of Bader et al. [8], empty circles and squares, experiments for proton and deuteron impact from Ref. [9]. Theories calculated considering the GII model. In general, throughout this article, solid and dotted lines denote CDWEIS and Born calculations, respectively. Contributions of $\mathrm{F}^{-}$(red lines) and $\mathrm{Li}^{+}$ions (blue lines), as indicated in the text. Thick solid and dotted green lines, total stopping power results per LiF molecule evaluated with the CDWEIS and Born approximations, respectively. (b) Inelastic cross sections for protons moving in a $\mathrm{LiF}$ crystal as a function of the incident velocity. Theoretical notation similar to (a).

the Madelung potential at ion sites moves up the binding energies of the bound states of the $\mathrm{Li}^{+}$cation and moves down the ones of the $\mathrm{F}^{-}$anion. Then, the influence of the grid changes the map of possible inelastic processes, making a new mechanism come to existence, that is, electronic transitions to $\mathrm{F}^{-}$excited states. These unoccupied excited states are the Frenkel excitons, as they are called in the language of condensed matter [6,7].

In this work we improve the GII model by considering the influence of the Coulomb grid of the crystal. We approximate the potential generated by the grid on the active electron by considering 44 spherical shells centered on the parent ion, in which the Coulomb charges of the neighboring ions are assumed to be uniformly distributed. These newly dressed cations and anions are here called onions, becoming the basic unit, instead of the isolated ions, of the crystal lattice. In this way, the crystal is reformulated as a grid of independent onions (GIO), which displays excited states that will be identified as excitons. In Sec. II we calculate the eigenenergies and radial wave functions of the onions by solving the corresponding Schrödinger equation. Within the GIO model we calculate electron yields (ionization), probability of exciton formation and electronic stopping by employing traditional atomic collision approximations, such as the simple first Born approximation or the more sophisticated CDWEIS approach, both developed in Sec. III. In Sec. IV we deal with surface collisions under grazing incidence conditions, where experiments are possible. We calculate the number of excitons produced by grazing impact of protons on a LiF crystal, which is the central goal of this article, and found that this number varies between 7 and 30 excitons in our energy range of interest. Atomic units are used except where otherwise stated.

\section{THE ONION MODEL}

Let us consider a perfect cubic piece of LiF crystal centered in the lithium ion that will be chosen as the active ion. We assume that the crystal is formed by $11^{3}-1=1330$ punctual ions, without counting the one at the center. In addition, for the whole crystal to be neutral we have to use an appropriate caging (Evjen method) by considering a fraction of the ions at the edges. Then, if we regard one electron of the lithium ion at the center as the active electron, its wave function is the solution of the Hamiltonian

$$
H_{\mathrm{Li}^{+}}^{\text {(crystal) }}=H_{\mathrm{Li}^{2+}}+V_{G}^{-(\vec{r})}
$$

with

$$
H_{\mathrm{Li}^{2+}}=-\frac{1}{2} \nabla_{r}^{2}+V_{\mathrm{Li}^{2+}}(r),
$$

where $V_{\mathrm{Li}^{2}}$ is the central core potential (formed by the nucleus $\mathrm{Li}^{3+}$ and one passive electron) and $V_{G}^{-}$is the potential created by the whole grid.

By considering just the long distance Coulomb interaction of the grid ions, $V_{G}^{-}(r)$ reads

$$
V_{G}^{-}(\vec{r})=\sum_{\vec{n} \neq 0} g_{\vec{n}} \frac{(-1)^{n_{x}+n_{y}+n_{z}}}{\left|\vec{r}-\vec{R}_{\vec{n}}\right|},
$$

where

$$
\vec{R}_{\vec{n}}=\frac{a}{2} \vec{n}, \quad \vec{n}=\left(n_{x}, n_{x}, n_{x}\right),
$$

with $a$ the lattice parameter $(a=7.597$ for $\mathrm{LiF})$ and $n_{x}, n_{y}$, and $n_{z}$ integer numbers ranging from -5 to 5 . In Eq. (3), $\Sigma_{\vec{n} \neq 0}$ indicates the sum on $n_{x}, n_{y}$, and $n_{z}$ so that the term $\vec{n}=(0,0,0)$ is excluded because the central Coulomb charge, corresponding to the active ion, has already been taken into account in $V_{\mathrm{Li}^{2+}}(r)$. The sign minus in the superindex of $V_{G}^{-}$ has been included to express that the grid has a net negative unitary charge. In this way, far from the crystal the active electron sees a grid charge -1 plus a charge +2 from $V_{\mathrm{Li}^{2+}}$, which produces a net positive unitary charge. The factors $g_{\vec{n}}$ in Eq. (3) describe the Evjen caging, that is, $g_{\vec{n}}=1$ except for ions placed at the limiting surface $\left(g_{\vec{n}}=1 / 2\right)$, at an arista $\left(g_{\vec{n}}=1 / 4\right)$ or at a vertex $\left(g_{\vec{n}}=1 / 8\right)$. This caging warranties the charge neutrality of the complete crystal.

For $V_{\mathrm{Li}^{2+}}(r)$ we use a simple expression given by

$$
V_{\mathrm{Li}^{2+}}(r)=-\frac{2}{r}-\frac{1}{r} \sum_{k=1}^{3} Z_{k}\left(1+\alpha_{k} r\right) \exp \left(-\mu_{k} r\right),
$$

where the parameters $Z_{k}, \mu_{k}$, and $\alpha_{k}$ were adjusted by solving the radial Schrödinger equation associated with $H_{\mathrm{Li}^{2+}}$, requiring that the corresponding ground state $1 \mathrm{~s}$ have a binding 
TABLE I. Parameters corresponding to the potentials given by Eqs. (5) and (10), respectively.

\begin{tabular}{lcccc}
\hline \hline Potential & $V_{\mathrm{Li}^{2}(1 s)}$ & $V_{\mathrm{F}^{0}(2 p)}$ & $V_{\mathrm{F}^{0}(2 s)}$ & $V_{\mathrm{F}^{0}(1 s)}$ \\
\hline$Z_{1}$ & 0.0102 & 3.4724 & 3.0113 & 4.2896 \\
$Z_{2}$ & 0.1018 & 5.0348 & 5.3931 & 4.5012 \\
$Z_{3}$ & 0.8879 & 0.4928 & 0.5957 & 0.2092 \\
$\mu_{1}$ & 1.1992 & 1.0377 & 0.6875 & 0.6588 \\
$\mu_{2}$ & 2.4446 & 2.6037 & 2.7568 & 3.0297 \\
$\mu_{3}$ & 3.4710 & 27.456 & 6.4830 & 12.003 \\
$\alpha_{1}$ & -0.0596 & -0.0149 & -0.0149 & -0.0050 \\
$\alpha_{2}$ & -0.0348 & 0.0050 & 0.0099 & -0.0033 \\
$\alpha_{3}$ & -0.0249 & -0.0967 & -0.0083 & -0.0672 \\
\hline \hline
\end{tabular}

energy $E_{1 s}$ and mean radial values $\langle r\rangle_{1 s}$ and $\langle 1 / r\rangle_{1 s}$ as close as possible to the values obtained by using the Hartree-Fock method [10], which are employed as a starting point. In all these cases, the Hartree-Fock eigenenergy was obtained with at least four significant figures, while the mean radial values were derived with a relative error of less than $1 \%$. The parameters $Z_{k}, \mu_{k}$, and $\alpha_{k}$ are listed in Table I for all the ions and states studied in this article. Figure 2(a) displays the potential $V_{\mathrm{Li}^{2+}}$ as a function of the radial distance $r$.

Now we proceed to assume that all the ions placed at a distance $R_{n}=\left|\vec{R}_{n}\right|=|\vec{n}| a / 2$ from the center form part of a spherical shell with a uniform superficial density of charge $\sigma_{n}=Q_{n} /\left(4 \pi R_{n}^{2}\right)$, where $Q_{n}$ is the net Coulomb charge of the ions spread uniformly upon the shell, taking into account of course the Evjen factors. Under this assumption the first shell, composed by six negative charges, is situated at $R_{1}=a / 2=$ 3.798 a.u., while the farthest one is situated at the diagonal, that is, $R_{44}=\sqrt{75} a / 2=32.89$ a.u., and encircles the 8 vertex, producing a net charge equal to -1 (recall that the charge at the vertexes is $\left.g_{( \pm 5, \pm 5, \pm 5)}=1 / 8\right)$. A list of the values of $R_{n}$ and $Q_{n}$ for the 44 shells considered in this article is displayed in Table II. The potential created by all the uniformly charged shells can easily be calculated to give rise to our onion potential $V_{O}^{-}(r)$, which is a central approximation to $V_{G}^{-}(\vec{r})$. It reads

$$
V_{O}^{-}(r)=-\sum_{n=1}^{44} Q_{n}\left[\frac{\Theta\left(R_{n}-r\right)}{R_{n}}+\frac{\Theta\left(r-R_{n}\right)}{r}\right],
$$

where $\Theta$ is the unitary Heaviside (step) function. The potential $V_{O}^{-}$given by Eq. (6) can be considered as the radial version of the Madelung potential and it represents the main hypothesis of the present work. Within this onion model, the Madelung potential at the origin is $V_{M}=V_{O}^{-}(r=0)=0.46005$ a.u. $=$ $12.51 \mathrm{eV}$, which is very close to the precise result $0.46037 \mathrm{a}$.u. of Ref. [19]. In Fig. 2(a) we also plot $V_{O}^{-}$as a function of the distance $r$ along the extreme values $R_{1}$ and $R_{44}$ of the shell radius, which are indicated with arrows. It is very important to verify that

$$
V_{\mathrm{Li}^{2+}}(r)+V_{O}^{-}(r) \underset{r \rightarrow \infty}{\sim}-\frac{2}{r}+\frac{1}{r}=-\frac{1}{r},
$$

which means that the active electron feels asymptotically the interaction of a unitary Coulomb charge. In the Wannier model this asymptotic potential is interpreted as that produced by the hole left behind by the excited electron.

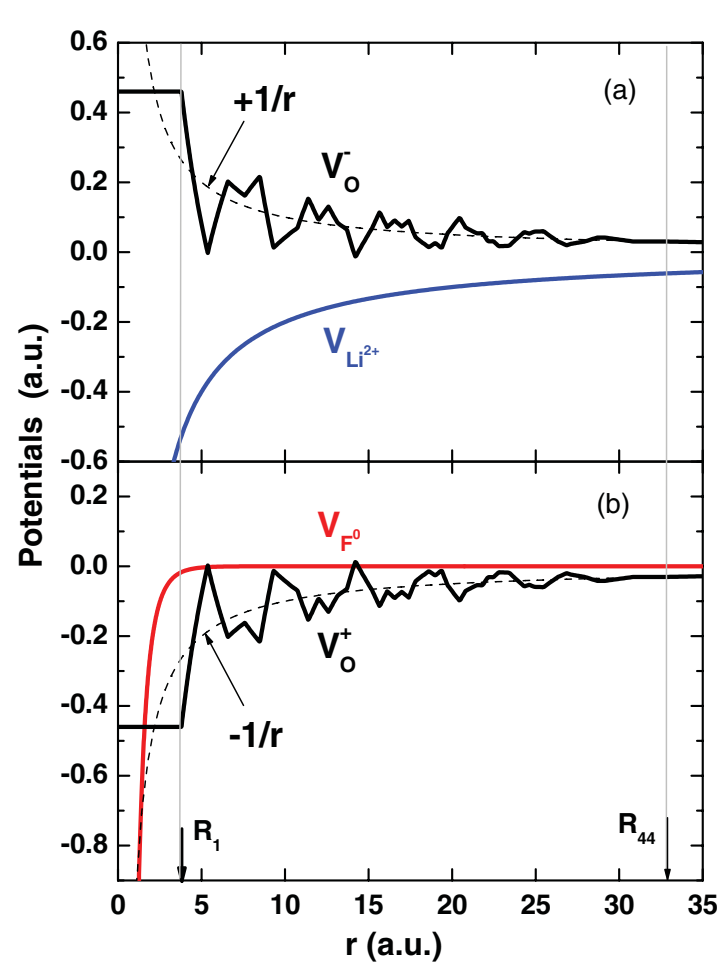

FIG. 2. (Color online) (a) Central ionic $V_{\mathrm{Li}^{2+}}$ and onion $\mathrm{V}_{O}^{-}$ potentials as a function of the distance $r$ to the nucleus. (b) Central $V_{\mathrm{F} 0}$ and onion $\mathrm{V}_{O}^{+}$potentials as a function of the distance $r$ to the nucleus. $\mathrm{R}_{1}$ and $\mathrm{R}_{44}$ indicates the position of the first and last onion shells, respectively.

To be coherent with the high energy approximations that we will use, in the potential of Eq. (7) we do not consider the screening of the medium via a dielectric constant. At high impact velocities, the impulse approximation considers that the electronic transition takes place at very short times, shorter than the relaxation time of the electronic cloud. For the same reasons we neglect the induced polarization due to the hole created by the electronic excitation, whose contribution has been largely referenced in the literature as the Mott-Littleton relaxation energy. This additional term would increases the energy by amount of $1.66 \mathrm{eV}(2.64 \mathrm{eV})$ if the hole is at anion (cation) site [20]. Anyway, the inclusion of a dielectric constant in our formalism would be straightforward, if necessary.

In Eq. (1), replacing $V_{G}^{-}(\vec{r})$ with the radial approximation $V_{O}^{-}(r)$ of Eq. (6) the problem is reduced to the calculation of a central potential, that is,

$$
H_{\mathrm{Li}^{+}}^{\text {(crystal) }} \simeq H_{\mathrm{Li}^{+} @}=-\frac{1}{2} \nabla_{r}^{2}+V_{\mathrm{Li}^{2+}}(r)+V_{O}^{-}(r),
$$

where we have resorted to the symbol @ to represent the Li ion embedded in the crystal grid, much like the use of @ to describe atoms within fullerenes (in fact, the idea is essentially the same). From Eq. (8), eigenfunctions and eigenenergies of the Hamiltonian $H_{\mathrm{Li}^{+}} @$ can be numerically obtained. To that end we used the RADIALF program developed by Salvat et al. [21]. Due to abrupt oscillations of $V_{O}^{-}(r)$, the numerical grid in the calculation was required to be very dense; about 5000 pivots were used to achieve a good convergence. The onion wave functions for the principal quantum numbers $n=1,2$, and 3 are plotted in Fig. 3 and the corresponding binding energies 
TABLE II. Shell radius $R_{n}$ and shell charges at the bulk $Q_{n}$ and at the surface $Q_{n}^{(s)}$ for the onion potential given by Eq. (6).

\begin{tabular}{|c|c|c|c|}
\hline$n$ & $R_{n}$ & $Q_{n}$ & $Q_{n}^{(s)}$ \\
\hline 1 & 3.7985 & -6 & -5 \\
\hline 2 & 5.3718 & +12 & +8 \\
\hline 3 & 6.5791 & -8 & -4 \\
\hline 4 & 7.5970 & +6 & +5 \\
\hline 5 & 8.4937 & -24 & -16 \\
\hline 6 & 9.3043 & +24 & +12 \\
\hline 7 & 10.743 & +12 & +8 \\
\hline 8 & 11.395 & -30 & -17 \\
\hline 9 & 12.011 & +24 & +16 \\
\hline 10 & 12.598 & -24 & -12 \\
\hline 11 & 13.158 & +8 & +4 \\
\hline 12 & 13.695 & -24 & -16 \\
\hline 13 & 14.212 & +48 & +24 \\
\hline 14 & 15.194 & +6 & +5 \\
\hline 15 & 15.661 & -48 & -28 \\
\hline 16 & 16.115 & +36 & +20 \\
\hline 17 & 16.557 & -24 & -12 \\
\hline 18 & 16.987 & +24 & +16 \\
\hline 19 & 17.406 & -48 & -24 \\
\hline 20 & 17.816 & +24 & +12 \\
\hline 21 & 18.608 & +24 & +12 \\
\hline 22 & 18.992 & -27 & -18 \\
\hline 23 & 19.368 & +60 & +32 \\
\hline 24 & 19.737 & -20 & -10 \\
\hline 25 & 20.455 & -60 & -32 \\
\hline 26 & 20.805 & +24 & +12 \\
\hline 27 & 21.487 & +12 & +8 \\
\hline 28 & 21.820 & -36 & -18 \\
\hline 29 & 22.148 & +36 & +20 \\
\hline 30 & 22.472 & -24 & -12 \\
\hline 31 & 22.791 & +24 & +12 \\
\hline 32 & 23.415 & +24 & +12 \\
\hline 33 & 24.322 & -36 & -20 \\
\hline 34 & 24.617 & +24 & +12 \\
\hline 35 & 24.908 & -12 & -6 \\
\hline 36 & 25.481 & -24 & -12 \\
\hline 37 & 26.316 & +8 & +4 \\
\hline 38 & 26.859 & +27 & +14 \\
\hline 39 & 27.126 & -6 & -3 \\
\hline 40 & 27.913 & +6 & +3 \\
\hline 41 & 28.678 & -12 & -6 \\
\hline 42 & 29.176 & -6 & -3 \\
\hline 43 & 30.859 & +6 & +3 \\
\hline 44 & 32.896 & -1 & -0.5 \\
\hline
\end{tabular}

are listed in Table III. To indicate that we are considering a particular $n l$ state of a dressing cation (onion) we denote it as $\mathrm{Li}^{+} @\left(1 s^{-1} \mid n l\right)$. The notation $1 s^{-1}$ indicates that the active electron was initially in the $1 s$ state, where the hole remains. Although for $\mathrm{Li}^{+} @$ the notation is redundant, for $\mathrm{F}^{-} @$ it will be necessary since the state $\mathrm{F}^{-} @\left(2 p^{-1} \mid n l\right)$ is different from $\mathrm{F}^{-} @\left(2 s^{-1} \mid n l\right)$.

The first important point is that $V_{O}^{-}(r)$ makes the binding energy of $\mathrm{Li}^{+} @\left(1 s^{-1} \mid 1 s\right)$ to increase by an amount equal to the Madelung constant, as compared with the one of the isolated ion, that is, $-75.98+12.51 \cong-63.46 \mathrm{eV}$ (see Table III). Furthermore, due to the asymptotic limit given by

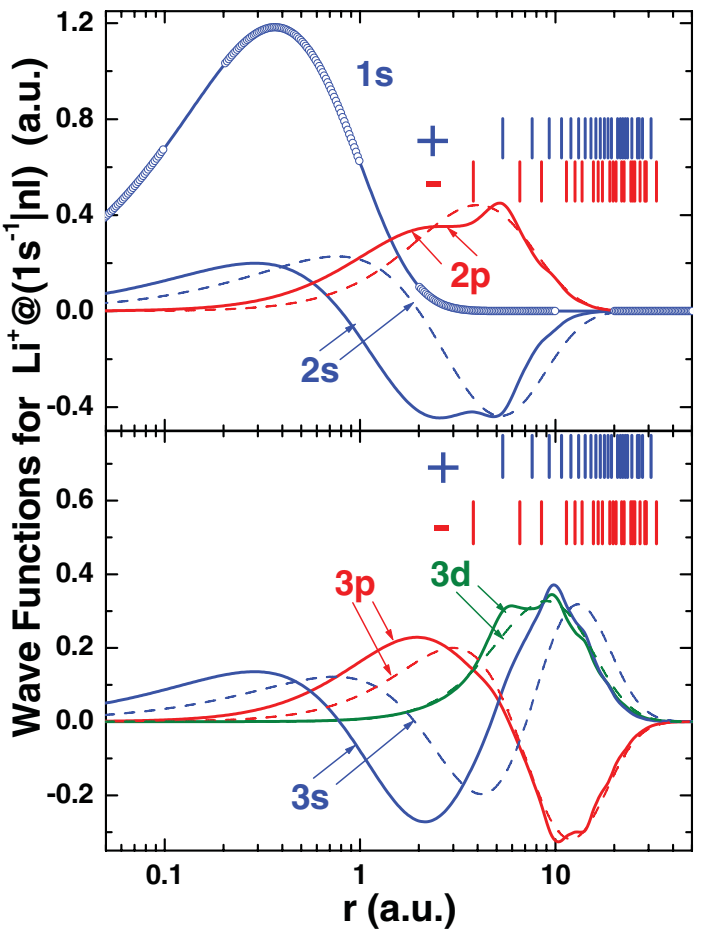

FIG. 3. (Color online) Wave functions, as a function of the distance $r$ to the nucleus. Solid lines, onion $\mathrm{Li}^{+} @$ wave functions; small empty circles, wave functions of the isolated $\mathrm{Li}^{+}$ion, as reported by Clementi and Roetti [10] (we have segmented this small circle line to avoid a full overlap with the solid line). Dashed lines, hydrogen wave functions. Vertical lines indicate the position of the positive + (blue) and negative - (red) onion shells.

Eq. (7), as $n$ increases the effect of $V_{O}^{-}(r)$ transforms the $\mathrm{He}^{2+}$-type structure of $V_{\mathrm{Li}^{2+}}$, characterized by energies close to $-2^{2} / 2 n^{2}$, into a hydrogen electronic series, characterized by eigenenergies similar to $-1^{2} / 2 n^{2}$. For the $1 s$ ground state the onion wave function is almost equal to the one of the isolated ion given by Clementi and Roetti [10]. However, for excited states the onion shells leave substantial footprints on the wave functions: the electronic density tends to depart from the negative shells (composed by $\mathrm{F}^{-}$anions), and to be attracted toward the positive ones (composed by $\mathrm{Li}^{+}$cations). As the angular momentum $l$ increases the onion wave functions approach the hydrogen ones, but it still bears some scars of the onion shells. For example, for $\mathrm{Li}^{+} @\left(1 s^{-1} \mid 3 d\right)$ the binding energy is $-1.61 \mathrm{eV}$, quite close to the value corresponding to $\mathrm{H}(3 d)$, which is $-1.51 \mathrm{eV}$.

In a way similar to the onion model for the active $\mathrm{Li}^{+}$ cation, we can derive an onion treatment for an active electron belonging to a $\mathrm{F}^{-}$anion situated now at the center of the grid. The corresponding Hamiltonian reads

$$
H_{\mathrm{F}^{-}}^{(\text {crystal })}=H_{\mathrm{F}^{0}}+V_{G}^{+}(\vec{r}),
$$

with

$$
H_{\mathrm{F}^{0}}=-\frac{1}{2} \nabla_{r}^{2}+V_{\mathrm{F}^{0}}(r),
$$

where $V_{G}^{+}(\vec{r})=-V_{G}^{-}(\vec{r})$ and the sign + indicates that the grid has now a net charge equal to +1 , that is, $V_{G}^{+}$behaves as an 
TABLE III. Energies of the bound states (in eV) for lithum and flouride ions as derived with the GII and the GIO models. $\Delta \epsilon_{j}, j=1,4$, energy difference with respect to the level $\mathrm{Li}^{+} @\left(1 s^{-1} \mid 1 s\right)(j=1)$, $\mathrm{F}^{-} @\left(2 p^{-1} \mid 2 p\right)(j=2), \mathrm{F}^{-} @\left(2 s^{-1} \mid 2 s\right)(j=3)$, and $\mathrm{F}^{-} @\left(1 s^{-1} \mid 1 s\right)(j=4)$, respectively. $\Delta \epsilon_{12}$, energy difference between the first excited state of $\mathrm{Li}^{0} @(2 s)$ and its nearest neighbor $\mathrm{F}^{-} @\left(2 p^{-1} \mid 2 p\right)$.

\begin{tabular}{lcc}
\hline \hline GII state & GIO state & Difference \\
\hline $\mathrm{Li}^{+}\left(1 s^{-1} \mid 3 p\right)=-6.14$ & $\mathrm{Li}^{+} @\left(1 s^{-1} \mid 3 p\right)=-1.58$ & $\Delta \epsilon_{1}=61.88$ \\
$\mathrm{Li}^{+}\left(1 s^{-1} \mid 3 d\right)=-6.05$ & $\mathrm{Li}^{+} @\left(1 s^{-1} \mid 3 d\right)=-1.61$ & $\Delta \epsilon_{1}=61.85$ \\
$\mathrm{Li}^{+}\left(1 s^{-1} \mid 3 s\right)=-6.59$ & $\mathrm{Li}^{+} @\left(1 s^{-1} \mid 3 s\right)=-2.00$ & $\Delta \epsilon_{1}=61.46$ \\
$\mathrm{Li}^{+}\left(1 s^{-1} \mid 2 p\right)=-13.92$ & $\mathrm{Li}^{+} @\left(1 s^{-1} \mid 2 p\right)=-3.57$ & $\Delta \epsilon_{1}=59.88$ \\
$\mathrm{Li}^{+}\left(1 s^{-1} \mid 2 s\right)=-15.57$ & $\mathrm{Li}^{+} @\left(1 s^{-1} \mid 2 s\right)=-5.00$ & $\Delta \epsilon_{1}=58.46$ \\
$\mathrm{Li}^{+}\left(1 s^{-1} \mid 1 s\right)=-75.98$ & $\mathrm{Li}^{+} @\left(1 s^{-1} \mid 1 s\right)=-63.46$ & $\Delta \epsilon_{1}=0.00$ \\
$\mathrm{~F}^{-}\left(2 p^{-1} \mid 3 d\right) \nexists$ & $\mathrm{F}^{-} @\left(2 p^{-1} \mid 3 d\right)=-1.69$ & $\Delta \epsilon_{2}=15.7$ \\
$\mathrm{~F}^{-}\left(2 p^{-1} \mid 3 p\right) \nexists$ & $\mathrm{F}^{-} @\left(2 p^{-1} \mid 3 p\right)=-3.49$ & $\Delta \epsilon_{2}=13.9$ \\
$\mathrm{~F}^{-}\left(2 p^{-1} \mid 3 s\right) \nexists$ & $\mathrm{F}^{-} @\left(2 p^{-1} \mid 3 s\right)=-6.28$ & $\Delta \epsilon_{2}=11.1$ \\
$\mathrm{~F}^{-}\left(2 p^{-1} \mid 2 p\right)=-4.92$ & $\mathrm{~F}^{-} @\left(2 p^{-1} \mid 2 p\right)=-17.38$ & $\Delta \epsilon_{2}=0.0$ \\
$\mathrm{~F}^{-}\left(2 s^{-1} \mid 3 d\right) \nexists$ & $\mathrm{F}^{-} @\left(2 s^{-1} \mid 3 d\right)=-2.19$ & $\Delta \epsilon_{3}=39.56$ \\
$\mathrm{~F}^{-}\left(2 s^{-1} \mid 3 p\right) \nexists$ & $\mathrm{F}^{-} @\left(2 s^{-1} \mid 3 p\right)=-4.85$ & $\Delta \epsilon_{3}=36.89$ \\
$\mathrm{~F}^{-}\left(2 s^{-1} \mid 3 s\right) \nexists$ & $\mathrm{F}^{-} @\left(2 s^{-1} \mid 3 s\right)=-8.22$ & $\Delta \epsilon_{3}=33.52$ \\
$\mathrm{~F}^{-}\left(2 s^{-1} \mid 2 s\right)=-29.22$ & $\mathrm{~F}^{-} @\left(2 s^{-1} \mid 2 s\right)=-41.75$ & $\Delta \epsilon_{3}=0.00$ \\
$\mathrm{~F}^{-}\left(1 s^{-1} \mid 3 d\right) \nexists$ & $\mathrm{F}^{-} @\left(1 s^{-1} \mid 3 d\right)=-3.71$ & $\Delta \epsilon_{4}=711.6$ \\
$\mathrm{~F}^{-}\left(1 s^{-1} \mid 3 p\right) \nexists$ & $\mathrm{F}^{-} @\left(1 s^{-1} \mid 3 p\right)=-8.07$ & $\Delta \epsilon_{4}=707.2$ \\
$\mathrm{~F}^{-}\left(1 s^{-1} \mid 3 s\right) \nexists$ & $\mathrm{F}^{-} @\left(1 s^{-1} \mid 3 s\right)=-11.6$ & $\Delta \epsilon_{4}=703.7$ \\
$\mathrm{~F}^{-}\left(1 s^{-1} \mid 1 s\right)=-702.56$ & $\mathrm{~F}^{-} @\left(1 s^{-1} \mid 1 s\right)=-715.3$ & $\Delta \epsilon_{4}=0.0$ \\
$\mathrm{Li}^{0}\left(1 s^{2} \mid 2 s\right)=-5.30$ & $\mathrm{Li}^{0} @\left(1 s^{2} \mid 2 s\right)=-3.97$ & $\Delta \epsilon_{12}=12.08$ \\
$\mathrm{Li}^{0}\left(1 s^{2} \mid 2 p\right)=-3.46$ & $\mathrm{Li}^{0} @\left(1 s^{2} \mid 2 p\right)=-2.93$ & $\Delta \epsilon_{12}=13.92$ \\
\hline \hline
\end{tabular}

attractive potential at long distances. We use the same potential structure of Eq. (5)

$$
V_{\mathrm{F}^{0}}(r)=\frac{0}{r}-\frac{1}{r} \sum_{k=1}^{3} Z_{k}\left(1+\alpha_{k} r\right) \exp \left(-\mu_{k} r\right),
$$

which is the central potential of the $\mathrm{F}^{-}$core (formed by the nucleus and nine passive electrons). The parameters $Z_{k}, \mu_{k}$, and $\alpha_{k}$ are obtained in similar way to the $\mathrm{Li}^{+}$case, being also displayed in Table I for the three occupied states of the $\mathrm{F}^{-}$. Note that as we are dealing with negative ions, $V_{\mathrm{F}^{0}}(r)$ presents a neutral asymptotic Coulomb charge. Again, by approximating $V_{G}^{+}(\vec{r}) \cong V_{O}^{+}(r)=-V_{O}^{-}(r)$ we derive the onion Hamiltonian

$$
H_{\mathrm{F}^{-}}^{\text {(crystal) }} \simeq H_{\mathrm{F}^{-} @}=-\frac{1}{2} \nabla_{r}^{2}+V_{\mathrm{F}^{0}}(r)+V_{O}^{+}(r),
$$

whose eigenfunctions can be numerically obtained and the corresponding eigenenergies are shown in Table III. In Fig. 2(b) we plot the potentials $V_{\mathrm{F}^{0}}(r)$ and $V_{O}^{-}(r)$ as a function of the radial distance $r$. It is interesting to note that once more the Coulomb grid makes the electron feel a central attractive potential, which behaves as

$$
V_{\mathrm{F}^{0}}(r)+V_{O}^{+}(r) \underset{r \rightarrow \infty}{\sim} \frac{0}{r}-\frac{1}{r}=-\frac{1}{r}
$$

at large distances. Consequently, even though there is no excited state for the isolated $\mathrm{F}^{-}$ion, there are infinite possible excited states when the anion is embedded in the grid. These excited states tend to be hydrogenic as the principal quantum number $n$ increases. Onion wave functions corresponding to the $\mathrm{F}^{-} @\left(2 p^{-1} \mid n l\right)$ states are plotted in Fig. 4, the features to be noted being very similar to those of the $\mathrm{Li}^{+}$case.
For the occupied states the onion potential leaves almost no footprints on the wave functions; the only trace is that the wave function of the state $\mathrm{F}^{-} @\left(2 p^{-1} \mid 2 p\right)$ decays slightly faster than the one of the isolated ion $\mathrm{F}^{-}(2 p)$ due to the influence of the Madelung potential. But for unoccupied excited states we find the same trend as before: the larger the angular momentum the more hydrogenic the wave functions.

In order to test the proposed GIO model it is convenient to compare our ground-state ionization energies with $a b$ initio quantum chemistry calculations [19,22,23]. For $\mathrm{F}^{-} @\left(2 p^{-1} \mid 2 p\right)$ we find an ionization energy of $17.4 \mathrm{eV}$, in contrast with the value $15.2 \mathrm{eV}$ obtained by Tatewaki and Miyoshi [19] using a $\mathrm{Li}_{14}{ }^{+} \mathrm{F}_{13}{ }^{-}$perfect cluster embedded in an ion cage and including correlation. This difference of $2.2 \mathrm{eV}$ between the ionization energies can be reduced by considering different starting values to determine the parameters of $V_{\mathrm{F}^{0}}$ as given by Eq. (10) [24]. By using a $\mathrm{Li}_{146}{ }^{+} \mathrm{F}_{63}{ }^{-}$cluster, Wirtz et al. [22] reported two peaks in the density of states, at 15.2 and $16.8 \mathrm{eV}$ respectively, with a total bandwidth of about $3.7 \mathrm{eV}$. However, experimental ionization thresholds are much lower than the theoretical results, ranging between 9.8 and $13 \mathrm{eV}$ [23]. Tatewaki [23] attributes this difference to the presence of defects which inevitably contaminate real crystals. By inserting defects at $\mathrm{Li}^{+}$sites he found a reasonable agreement with the experiments. If we let our Coulomb grid points be polarized, we would obtain an increase in energy of about $1.66 \mathrm{eV}$ due to the Mott-Littleton relaxation effect [20].

Another important magnitude to compare is the difference of energy associated with the electronic transition from an $\mathrm{F}^{-} @$ anion to the nearest neighbor $\mathrm{Li}^{+} @$ cation (charge 


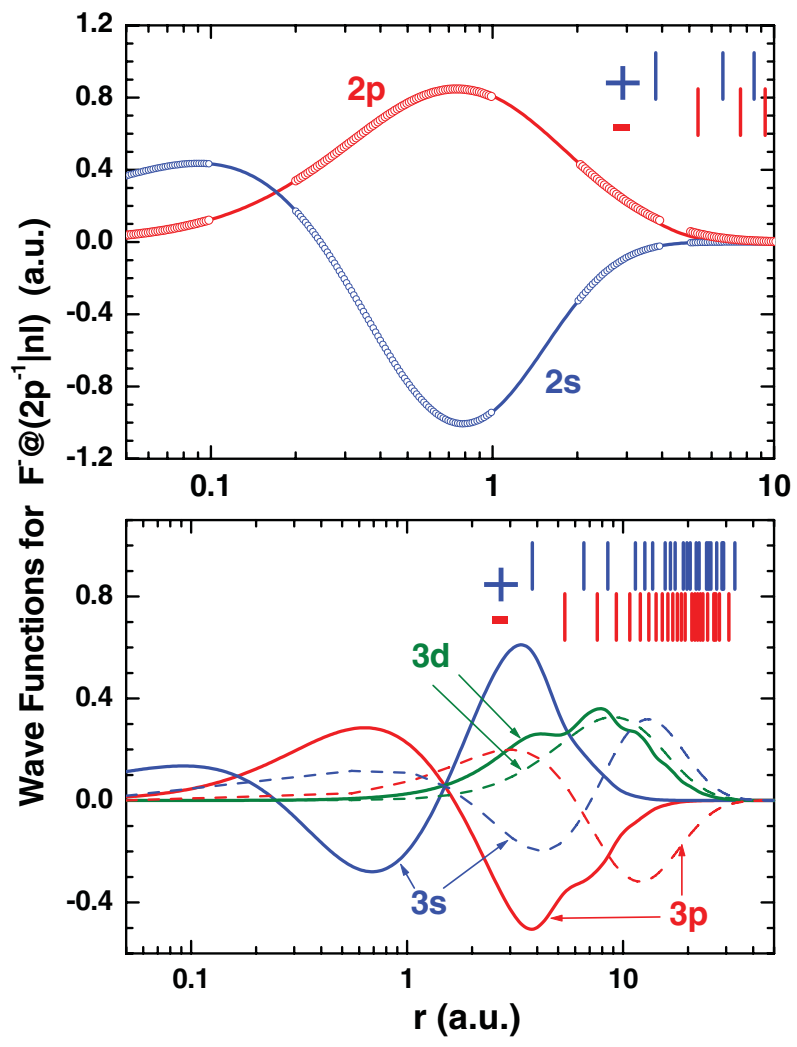

FIG. 4. (Color online) Wave functions as a function of the distance $r$ to the nucleus. Solid lines, onion $\mathrm{F}^{-} @$ wave functions; empty circles, wave functions of the isolated $\mathrm{F}^{-}$ion, as reported by Clementi and Roetti [10] (we have segmented this small circle line to avoid a full overlap with the solid line). Dashed lines, hydrogen wave functions. Vertical lines indicate the position of the positive + (blue) and negative - (red) onion shells.

transfer). The energy difference between the first excited state of $\mathrm{Li}^{0} @(2 s)$ and its nearest neighbor $\mathrm{F}^{-} @\left(2 p^{-1} \mid 2 p\right)$ is $\Delta \epsilon_{12}=12.08 \mathrm{eV}$ (see Table III). This value is similar to the energy $11.8 \mathrm{eV}$ of the first excited state reported in Ref. [19], which was identified as an electronic transition producing a localized hole at the central $\mathrm{F}^{-}$accompanied by an electronic density spanned by the $\mathrm{Li}(2 s)$ orbital.

Finally, the onion model can be extended from the bulk to the surface by drastically removing the ions of the hemisphere corresponding to the vacuum region. In the surface case, the number of crystal ions decreases to 725 and the values of the shell charges $Q_{n}^{(s)}$ are smaller than those of the bulk, as observed in Table II. For example, note that in the first shell corresponding to the nearest neighboring ions to a surface $\mathrm{Li}$ cation we have five negative ions, instead of the six in the bulk, because the ion above the surface is missing. The surface Madelung constant is slightly smaller than the bulk value $V_{M}^{(s)}=0.4425$ a.u. $=12.04 \mathrm{eV}$, indicating that there is a slight relaxation of the Madelung potential of about 0.0165 a.u. $=$ $0.45 \mathrm{eV}$ in the surface case.

\section{INELASTIC COLLISIONS IN THE BULK}

In this section we study different inelastic processes produced by fast protons moving in the bulk of a $\mathrm{LiF}$ crystal.
They will be classified in excitation or ionization transitions according to the final state of the active electron: bound or continuum state, respectively.

\section{A. Excitation transitions}

We consider two families of direct excitation processes produced by proton impact,

$$
\begin{aligned}
\mathrm{H}^{+}+\mathrm{Li}^{+} @\left(1 s^{-1} \mid 1 s\right) & \rightarrow \mathrm{H}^{+}+\mathrm{Li}^{+} @\left(1 s^{-1} \mid n l\right), \\
\mathrm{H}^{+}+\mathrm{F}^{-} @\left(i^{-1} \mid i\right) & \rightarrow \mathrm{H}^{+}+\mathrm{F}^{-} @\left(i^{-1} \mid n l\right),
\end{aligned}
$$

with $i=1 s, 2 s$, and $2 p$.

An important outcome of the GIO model is that both onion-electron systems, described by the Hamiltonians $H_{\mathrm{Li}^{+} @}$ and $H_{\mathrm{F}^{-}} @$, respectively, are neutral and with localized wave functions. In what follows we identify the necessary steps to reduce the extended tight-binding model to a local description.

Within the tight-binding formalism [25] the initial and final unperturbed electronic states $\phi_{\vec{\kappa}_{j}, j}(\vec{r})$ read

$$
\phi_{\vec{\kappa}_{j}, j}(\vec{r})=\sum_{\vec{R}} e^{i \vec{k}_{j} \cdot \vec{R}} \varphi_{\vec{\kappa}_{j}, j}(\vec{r}-\vec{R}), \quad j=i, f,
$$

where the wave vector $\vec{\kappa}_{j}$ has been introduced to identify a given crystal state within the band $j$, with $\vec{\kappa}_{j}$ belonging to the first Brioullin zone, and the sum involves all $\vec{R}$ positions of the target ions. The state $\varphi_{\vec{\kappa}_{j}, j}(\vec{r}-\vec{R})$ is the full Wannier function centered on the lattice site $\vec{R}$. We can approximate $\varphi_{\vec{k}_{j}, j}(\vec{r}-\vec{R})$ by the eigenfunctions of $H_{\mathrm{Li}^{+} @}$ or $H_{\mathrm{F}^{-} @}$ as appropriate [26]. In such a way, the GIO model ensembles neutral onions instead of the isolated ions considered within the GII model. The GIO model is expected to work better than the GII one because the Hamiltonians $H_{\mathrm{Li}^{+} @}$ and $H_{\mathrm{F}^{-} @}$ already include the Madelung potential, so the energy shift term $\beta$ of the tight-binding energy [25] should be smaller. In addition, as the wave function $\varphi_{i}$ of $\mathrm{F}^{-} @\left(2 p^{-1} \mid 2 p\right)$ decays slightly faster than the one of the isolated ion $\mathrm{F}^{-}(2 p)$, the overlap between nearest-neighbor Wannier functions is expected to be smaller as well. Structurally, the tight binding model for onions would resemble more a hybridization of neutral $\mathrm{Ne}$ and $\mathrm{He}$ than that of isolated ions.

Within the GIO model, the amplitude for the electronic transition $i \rightarrow f$ can be expressed as a coherent sum of individual electronic transitions from different lattice sites. In this work we neglect the electronic transitions that involve different lattice sites, that is, only electronic excitations in which the initial and final onion states correspond to the same lattice site are considered. After adding the contributions from the different crystal states by integrating $\vec{\kappa}_{i}$ and $\vec{\kappa}_{f}$ over the first Brioullin zones and considering the incoherent limit of such $\vec{\kappa}_{j}$ integrals [11], the excitation probability can be expressed in terms of the local $T$-matrix element $T_{i f}$ associated with the excitation between individual states $\varphi_{i}$ and $\varphi_{f}$, both corresponding to the same onion. Consequently, under these assumptions it is possible to reduce the tight-binding model to a local description involving one onion only.

The local $T$-matrix element is evaluated here using two different well-known distorted-wave methods, reading

$$
T_{i f}=\left\langle\chi_{f}\left|V_{f}^{\dagger}\right| \chi_{i}\right\rangle
$$


where $\chi_{i}$ and $\chi_{f}$ are the distorted wave functions in the entrance and exit channels, respectively, and $V_{f}$ represents the perturbative potential in the final channel.

If we are interested in high energy collisions we can directly resort to the first Born approximation, whose initial and final distorted-wave functions are the unperturbed ones, that is,

$$
\chi_{j}^{B}(\vec{R}, \vec{r})=\frac{\exp \left(i \vec{K}_{j} \cdot \vec{R}\right)}{(2 \pi)^{3 / 2}} \varphi_{j}(\vec{r}), \quad \text { with } \quad j=i, f,
$$

where $\vec{R}$ denotes the projectile position with respect to the center of mass of the active ion, $\vec{K}_{i}\left(\vec{K}_{f}\right)$ is the initial (final) projectile momentum vector, and $\varphi_{i}\left(\varphi_{f}\right)$ is the initial (final) electronic wave function, eigenfunction of $H_{\mathrm{Li}^{+} @}$ or $H_{\mathrm{F}^{-}}$. Then, within the first Born approach $T_{i f}$ is simply given by

$$
T_{i f}^{B}=\frac{1}{(2 \pi)^{3 / 2}} \widetilde{V}_{P}(p) F_{i f}(\vec{p}),
$$

where $\widetilde{V}_{P}(p)=-Z_{P} \sqrt{2 / \pi} / p^{2}$ is the Fourier transform of the projectile-electron Coulomb interaction, with $Z_{P}$ the projectile charge $\left(Z_{P}=1\right.$ for proton impact), and $\vec{p}$ is the momentum transfer vector. The function $F_{i f}$ represents the atomic form factor

$$
F_{i f}(\vec{p})=\int d \vec{r} \varphi_{f}^{*}(r) \exp (i \vec{p} \cdot \vec{r}) \varphi_{i}(r),
$$

which can be expressed as a series of Clebsch-Gordan coefficients (see Appendix for details).

The first Born approximation, given by Eq. (18), is a perturbative method, useful at large impact velocities only. But if we are interested in the intermediate energy regime we have to improve the calculation of the transition matrix. In this article we resort to the CDWEIS approximation, which was recently applied to describe total ionization with success [27]. Within the CDWEIS approach the distorted wave functions include the projectile distortion in the initial and final channels in the following way:

$$
\begin{aligned}
& \chi_{i}^{\mathrm{EIS}}(\vec{R}, \vec{r})=E_{i}^{+}\left(\vec{r}_{P}\right) \chi_{i}^{B}(\vec{R}, \vec{r}), \\
& \chi_{f}^{\mathrm{CDW}}(\vec{R}, \vec{r})=D_{f}^{-}\left(\vec{r}_{P}\right) \chi_{f}^{B}(\vec{R}, \vec{r}),
\end{aligned}
$$

where

$$
\begin{gathered}
E_{i}^{+}\left(\vec{r}_{P}\right)=\exp \left[-i \xi \ln \left(v r_{P}+\vec{v} \cdot \vec{r}_{P}\right)\right], \\
D_{f}^{-}\left(\vec{r}_{P}\right)=N_{\xi 1} F_{1}\left(-i \xi, 1,-i v r_{P}-i \vec{v} \cdot \vec{r}_{P}\right), \\
N_{\xi}=\exp (\xi / 2) \Gamma(1+i \xi),
\end{gathered}
$$

with $\vec{v}$ the projectile velocity, $\xi=Z_{P} / v,{ }_{1} F_{1}$ the confluent hypergeometric, and $\vec{r}_{P}=\vec{r}-\vec{R}$. After some algebra, the CDWEIS $T$-matrix element can be expressed as

$$
T_{i f}^{\mathrm{CDWEIS}}=-\frac{1}{(2 \pi)^{3 / 2}} \vec{W}(\vec{p}) \cdot \vec{G}_{i f}(\vec{p}),
$$

where

$$
\begin{aligned}
\vec{G}_{f, i}(\vec{p}) & =\int d \vec{r}\left[\vec{\nabla} \varphi_{f}(\vec{r})\right]^{*} e_{i}^{i \vec{p} \cdot \vec{r}} \varphi_{i}(\vec{r}), \\
\vec{W}(\vec{p}) & =\int \frac{d \vec{r}}{(2 \pi)^{3 / 2}} E_{i}^{+}(\vec{r}) e^{-i \vec{p} \cdot \vec{r}} \vec{\nabla} D_{f}^{-*}(\vec{r}) .
\end{aligned}
$$

In the Appendix we summarize the expression of $\vec{G}_{i f}(\vec{p})$ in terms of radial integrals, while $\vec{W}(\vec{p})$ has a closed form in terms of the hypergeometric functions ${ }_{2} F_{1}$, reported in Ref. [28]. Notice that as both $\varphi_{i}$ and $\varphi_{f}$ are numerical solutions of the same unperturbed Hamiltonian $\left(H_{\mathrm{Li}^{+} @}\right.$ or $\left.H_{\mathrm{F}^{-}} @\right)$, the transition matrix $T_{i f}^{\text {CDWEIS }}$ does not display prior-post discrepancies [29], as we have here numerically verified.

From Eqs. (18) and (25), Born and CDWEIS T-matrix elements depend on the vector $\vec{p}$, which can be expressed as $\vec{p}=\left(\vec{\eta}, p_{m}\right)$, where $\vec{\eta}$ is the component of the momentum transfer perpendicular to the incident velocity $\vec{v}$ and $p_{m}$ is the minimum momentum transfer along the direction of $\vec{v}$, $p_{m}=\left(\varepsilon_{f}-\varepsilon_{i}\right) / v$, with $\varepsilon_{i}\left(\varepsilon_{f}\right)$ the initial (final) electron energy given in Table III. Then, the excitation cross section reads

$$
\sigma_{i f}^{(e)}=\frac{(2 \pi)^{4}}{v^{2}} \int d \vec{\eta}\left|T_{i f}\right|^{2}
$$

In Fig. 5 we plot the total excitation cross section $\sigma^{(e)}=$ $\sum_{i, f} \sigma_{i f}^{(e)}$, and the stopping power (energy loss per unit path length) $S^{(e)}=\sum_{i, f}\left(\varepsilon_{f}-\varepsilon_{i}\right) \sigma_{i f}^{(e)}$, evaluated within the Born and CDWEIS approximations, as a function of the incident velocity. For the onion $\mathrm{F}^{-} @$ we have considered the initial states $i=2 s$ and $2 p$ and the final excited states $f=3 l$ and $4 l$, while for $\mathrm{Li}^{+} @$ the considered initial and final orbitals are $i=1 s$ and $f=2 l$ and $3 l$, respectively, with $l$ the angular momentum number. In all the cases the contribution of excitation from the $F^{-} @\left(2 p^{-1} \mid 2 p\right)$ state is the most relevant.

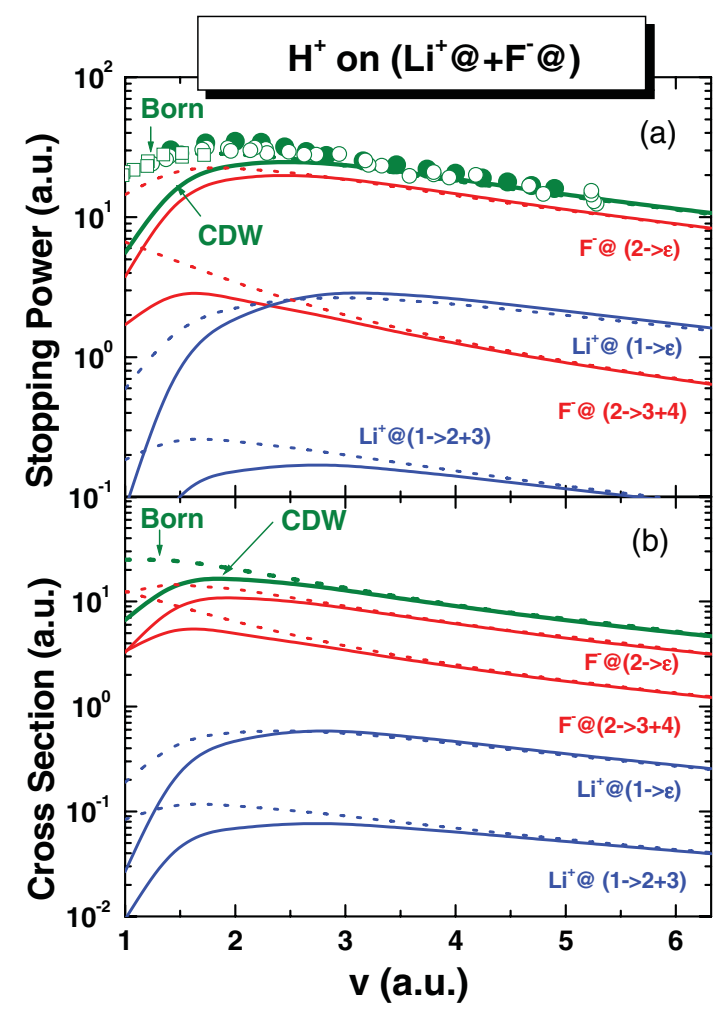

FIG. 5. (Color online) (a) Stopping power cross sections for protons moving in a $\mathrm{LiF}$ crystal, as a function of the proton velocity, considering the GIO model. Notation similar to Fig. 1. $\mathrm{F}^{-} @(2->3+4)$, excitation from the level $n_{i}=2$ to the levels $n_{f}=3$ and 4 of the fluoride onion. (b) Inelastic cross sections for protons moving in LiF crystal, as a function of the proton velocity, considering the GIO model. Theoretical notation similar to (a). 
As expected, at high impact energies CDWEIS results tend to the first order ones derived from the first Born approach, but substantial differences are observed at intermediate energies.

\section{B. Ionization transitions}

For protons moving inside a LiF crystal we consider two mechanisms of electron production,

$$
\begin{aligned}
\mathrm{H}^{+}+\mathrm{Li}^{+} @\left(1 s^{-1} \mid 1 s\right) & \rightarrow \mathrm{H}^{+}+\mathrm{Li}^{2+} @\left(1 s^{-1}\right)+e @, \\
\mathrm{H}^{+}+\mathrm{F}^{-} @\left(i^{-1} \mid i\right) & \rightarrow \mathrm{H}^{+}+\mathrm{F}^{0} @\left(i^{-1}\right)+e @,
\end{aligned}
$$

where the term $e @$ has been introduced to indicate that the electron continuum state is also embedded in the grid; that is, the electron ejected with momentum $\vec{k}$ is described by an eigenfunction $\varphi_{\vec{k}}^{-}(\vec{r})$ of the onion Hamiltonian $H_{\mathrm{Li}^{+} @ \text { or }}$ $H_{\mathrm{F}^{-}}$, with positive energy $\varepsilon=k^{2} / 2$ and outgoing asymptotic conditions. Then, the $T$-matrix element associated with the ionization process can be calculated in the same way as in the previous subsection [Eqs. (18) and (25)] but now using the unperturbed final state $\varphi_{f}(\vec{r})=\varphi_{\vec{k}}^{-}(\vec{r})$, which is again orthogonal to the initial state $\varphi_{i}(\vec{r})$. However, the evaluation of $T_{i f}$ gets much more complicated than in the excitation case since we have to expand $\varphi_{\vec{k}}^{-}(\vec{r})$ in different angular momenta (we used up to 24 values of $l$ ) and for each angular momentum $l$ a numerical integration is required to solve the radial integral. Besides, as we are dealing with ionization, to obtain the total cross section we need to evaluate two further integrals: on the electron energy we used a grid of about 50 pivots and on the ejection angle we used 28 angles.

Partial ionization cross section and stopping power evaluated within Born and CDWEIS approximations are displayed in Fig. 5, along with the excitation results. By far, ionization from $\mathrm{F}^{-} @\left(2 p^{-1} \mid 2 p\right)$ is the dominant mechanism, as also observed within the GII model (see Fig. 1). But the ionization contribution derived within the GIO formalism is substantially smaller than the GII one. This behavior is originated by the Madelung potential, which deepens the binding energy of the initial states by an amount close to the Madelung energy, thus reducing the ionization probability. Like in Fig. 1, in Fig. 5 we also display the total values of the inelastic cross section and stopping power as a function of the incident velocity. While the simple first Born approximation gives a good account of the experiments of stopping, including excitation processes, the CDWEIS theory, which should work better at intermediate velocities, does not introduce improvements, decreasing excessively in comparison with the experiments at the lower velocities.

On the other hand, the reason why both the GII and GIO models give about the same stopping power at high energies, as shown in Figs. 1 and 5, should be found in the f-sum rule, which imposes a closure relation regardless of the basis used. However, the GIO model involves a physical picture of the energy loss process different from the one of the GII model, taking into account the contribution of an additional mechanism. In the GII model no electron excitation of $\mathrm{F}^{-}$ is possible and the stopping is essentially due to the $\mathrm{F}^{-}$ ionization, as observed in Fig. 1. In turn, within the GIO model the ionization of the $\mathrm{F}^{-} @$ is lower than the GII value but $\mathrm{F}^{-} @$ excitation and $\mathrm{Li}^{+} @$ ionization acquire additional importance. Then we can say that the contributions of the different mechanisms are redistributed in the GIO model although the total stopping power remains the same as in the GII one at high energies. Notice that at $200 \mathrm{keV}$, the $\mathrm{F}^{-} @$ excitation contributes with $7 \%$ to the total stopping and with $27 \%$ to the total cross section. It means that one out of four inelastic transitions is an $\mathrm{F}^{-}$@ excitation.

\section{Interference structures}

In addition, an interesting feature that comes up when we use the GIO model is that interference effects appear in the single differential ionization cross section. In Figs. 6(a) and 6(b) we show CDWEIS differential cross sections for ionization from the onion states $\mathrm{F}^{-} @\left(2 p^{-1} \mid 2 p 0\right)$ and $\mathrm{Li}^{+} @\left(1 s^{-1} \mid 1 s\right)$, respectively, as a function of the electron energy $\varepsilon$ for $200 \mathrm{keV}$ and $1 \mathrm{MeV}$ protons. In the figures we have also included results derived by considering the isolated ion states $\mathrm{F}^{-}(2 p 0)$ and $\mathrm{Li}^{+}(1 s)$ (GII model). The interference structures observed in the onion spectra of Figs. 6(a) and 6(b) might be related to the coherent superposition of different quantum paths: the direct path and the ones that rebound at the different onion shells. This fact can clearly be appreciated by plotting the enhancement factor of the continuum onion wave function $\varphi_{\vec{k}}^{-}$, defined as its value at the origin $J(k)=\varphi_{\vec{k}}^{-}(r=0)$, which is related to the Jost function. The enhancement functions $J(k)$ corresponding to the final continuum states considered in Figs. 6(a) and 6(b) are shown in Figs. 6(c) and 6(d), respectively. By comparison we plot also the Coulomb enhancement factors $J_{c}(k)$ given by the well known expression

$$
J_{c}(k)=\frac{2 \pi \varsigma}{1-\exp (-2 \pi \varsigma)}, \quad \text { with } \quad \varsigma=\frac{Z}{k},
$$
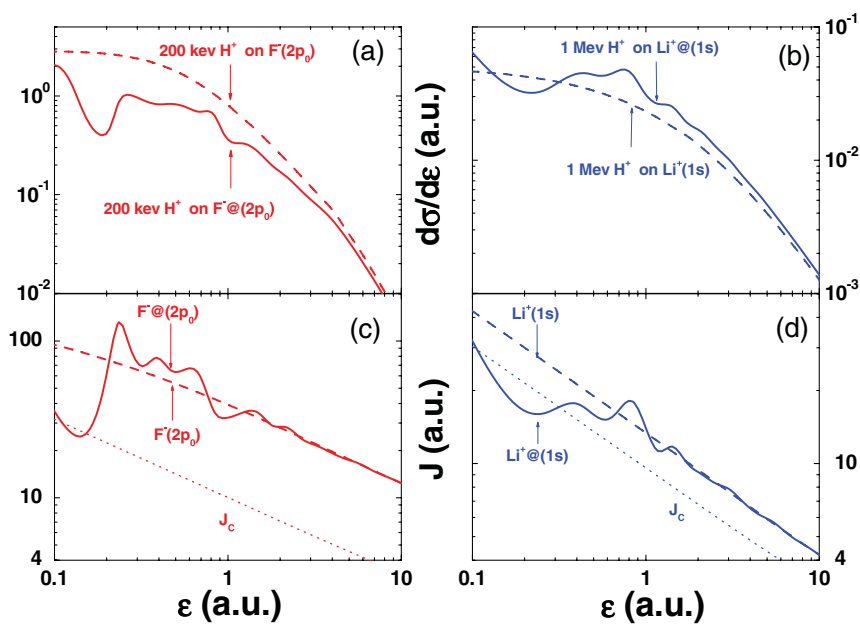

FIG. 6. (Color online) (a) Single differential ionization cross sections, as a function of the electron energy, for $200 \mathrm{keV}$ protons colliding with the anion $\mathrm{F}^{-}(2 p 0)$. Solid and dashed lines, CDWEIS results evaluated within the GIO and GII models, respectively. (b) Similar to (a) for $1 \mathrm{MeV}$ protons colliding with the cation $\mathrm{Li}^{+}(1 s)$. (c) Enhancement factors for $\mathrm{F}^{-} @(2 p 0)$ (solid line) and $\mathrm{F}^{-}(2 p 0)$ (dashed line), as a function of the electron energy, as explained in the text. $J_{c}$ Coulomb enhancement factor for $Z=2.26$ a.u., as given by Eq. (31). (d) Similar to (c) for the cation $\mathrm{Li}^{+}(1 s) . J_{c}$ Coulomb enhancement factor for $Z=2.16$ a.u., as given by Eq. (31). 
where the $Z$ values were chosen as $Z=2.26$ and 2.16 a.u. for $\mathrm{F}^{-} @$ and $\mathrm{Li}^{+} @$, respectively, in order to fit the binding energies of the states $\mathrm{F}^{-} @\left(2 p^{-1} \mid 2 p\right)$ and $\mathrm{Li}^{+} @\left(1 s^{-1} \mid 1 s\right)$ with the expression $Z^{2} /\left(2 n^{2}\right)$ (see Table III). The figures shows that while the Jost functions associated with the continuum states of the isolated ions, as well as the Coulombic ones $J_{c}(k)$ display monotonically decreasing behaviors, the Jost functions of the onion states $\mathrm{F}^{-} @\left(2 p^{-1} \mid \varepsilon\right)$ and $\mathrm{Li}^{+} @\left(1 s^{-1} \mid \varepsilon\right)$ present strong destructive and constructive interference regions as a function of the electron energy, which should be related to rebounds at the different shells.

\section{Exciton transmission velocity}

As mentioned in Sec. I, the formation of the exciton corresponds to the electronic excitation to an excited state of the onion $\mathrm{F}^{-} @$. We have reduced the exciton formation by collisions to a local process in which one lattice site is involved only, that is, the hole and the electron are placed on the same crystal ion and therefore we are dealing with Frenkel excitons [7]. After the exciton is created, one interesting question is to describe its movement. In this subsection we will estimate the velocity of propagation of the exciton by employing our picture. Within our model the movement of the exciton can be interpreted as an excitation transfer mechanism between different sites [30]. For the simplest case in which only two lattice sites (or onions) are involved, the dimer formed by two nearest neighbor $\mathrm{F}^{-} @$ onions includes a hole in the state $h$ and one electron in the state $e$, consequently the exciton transmission can be expressed as

$$
\begin{aligned}
& \mathrm{F}^{-} @\left(h^{-1} \mid e^{1}\right)+\mathrm{F}^{-} @\left(h^{1} \mid e^{0}\right) \\
& \quad \rightarrow \mathrm{F}^{-} @\left(h^{1} \mid e^{0}\right)+\mathrm{F}^{-} @\left(h^{-1} \mid e^{1}\right) .
\end{aligned}
$$

This mechanism then transfers the hole-excited-state $\left(h^{-1} \mid e^{1}\right)$ couple from one center to the other one without charge displacement. The basic velocity of transfer of the $\left(h^{-1} \mid e^{1}\right)$ couple from one center of the dimer to the other, which is situated at a distance $\vec{R}_{1}\left(R_{1}=5.37 \mathrm{a}\right.$.u. in our case $)$, is given by the expression $v_{h^{-1} \mid e}=R_{1} / \tau_{h^{-1} \mid e}$, where $\tau_{h^{-1} \mid e}$ is the transfer time given by [31]

$$
\frac{1}{\tau_{h^{-1} \mid e^{1}}}=\kappa \frac{\pi}{2 R_{1}^{3}}\left[\left|\langle\vec{r}\rangle_{h, e}\right|^{2}-3\left|\widehat{R}_{1} \cdot\langle\vec{r}\rangle_{h, e}\right|^{2}\right],
$$

where the dipole interaction element is

$$
\langle\vec{r}\rangle_{h, e}=\int d \vec{r} \varphi_{h}^{*}(\vec{r}) \vec{r} \varphi_{e}(\vec{r})
$$

and the factor $\kappa$ accounts for the crystal structure $(\kappa=1$ for the dimer). Note that Eq. (33) can be obtained from the simplified form of the Bethe-Salpeter equation \{see, for example, Eq. (3) of Ref. [32] \} by approximating the interaction by its dipoledipole large-distance limit between Frenkel excitons (localized in the same center). Using our onion states to calculate the dipole elements, it produces for $\kappa=1: v_{2 p_{z}^{-1} \mid 3 s}=0.018$ a.u, $v_{2 p_{x}^{-1} \mid 3 s}=0.0059$ a.u., and $v_{2 s^{-1} \mid 3 p}=0.00021$ a.u., where $\widehat{z}$ was chosen along $\widehat{R}_{1}$.

It is interesting to compare these values with the hole velocities in which an unit charge $e$ moves from one site to its neighbor. In our onion formalism the transmission of one hole alone can be expressed as

$$
\mathrm{F}^{-} @\left(h^{-1}\right)+\mathrm{F}^{-} @\left(h^{1}\right) \rightarrow \mathrm{F}^{-} @\left(h^{1}\right)+\mathrm{F}^{-} @\left(h^{-1}\right) .
$$

The hole velocity transmission is again given by $v_{h^{-1}}=$ $R_{1} / \tau_{h^{-1}}$, where now

$$
\frac{1}{\tau_{h^{-1}}}=\kappa \frac{\pi}{2 R_{1}^{3}} \int d \vec{r} \varphi_{h}(\vec{r}) V_{h}(\vec{r}) \varphi_{h}\left(\vec{r}-\vec{R}_{1}\right)
$$

with $\varphi_{h}(\vec{r})$ the wave function of the $h$ state and $V_{h}$ its central potential. Using our onion states and potentials, we obtain for $\kappa=1$ the following hole velocities: $v_{2 p_{z}^{-1}}=0.126$ a.u., $v_{2 p_{x}^{-1}}=0.024$ a.u., and $v_{2 s^{-1}}=0.016$ a.u. For a real $\mathrm{LiF}$ crystal, just to make an idea, we can consider the first 12 neighbors (noninteracting themselves), so $\kappa \cong \sqrt{12}$. Then we obtain $v_{2 p^{-1}} \simeq \sqrt{12}\left(v_{2 p_{z}^{-1}}+2 v_{2 p_{x}^{-1}}\right) / 3 \simeq 0.2$ a.u. This value is in between the velocities of hot holes $\left(v_{h^{-1}}=0.3\right.$ a.u.) and cold holes $\left(v_{h^{-1}}=0.1\right.$ a.u.) used by Wirtz et al. [33].

Note that as we are dealing with high impact velocities, we consider that the hole created in the target travels at a velocity much lower that the projectile one, and so a frozen crystal model is reasonable. This is not the case if we deal with low velocity projectiles [3] where the hole mobility is a variable to be taken into consideration. Furthermore, electron capture could be rethought as precisely a hole migration from the projectile to a target site.

\section{GRAZING EXCITATIONS}

In this section we deal with the production of excitons by grazing collisions of swift protons with LiF surfaces. As seen in Sec. III, the transition probability from the initial band $i$ [11] in the incoherent limit (after integrating over the crystal wave vector $\vec{\kappa}_{i}$ and $\vec{\kappa}_{f}$ ) coincides with that derived by assuming the crystal surface as composed by a continuous distribution of onions. Furthermore, taking into account that in the energy range of interest the projectile movement can be described in terms of a classical trajectory, the transition probability for a given projectile path is expressed as

$$
P_{i f}=\delta_{s} \int_{-\infty}^{\infty} d x \int_{-\infty}^{\infty} d y\left|A_{i f}[\vec{b}(x, y)]\right|^{2},
$$

where $A_{i f}(\vec{b})$ is the transition amplitude corresponding to the surface onion placed at the position $(x, y)$ on the surface plane, as a function of the impact parameter $\vec{b}$, and $\delta_{s}$ is the surface density of crystal ions. In Eq. (37) the impact parameter $\vec{b}$ depends on both, the target position and the classical projectile path. Within the GIO model, the transition amplitude $A_{i f}$ can be obtained from the CDWEIS $T$-matrix element given by Eq. (25) by using the well known eikonal approximation; that is,

$$
A_{i f}(\vec{b})=\frac{2 \pi}{v} \int d \vec{\eta} \exp (i \vec{\eta} \cdot \vec{b}) T_{i f}^{\mathrm{CDWEIS}},
$$

where $\vec{\eta}$ is the perpendicular momentum transfer, as defined in Eq. (28). Calculations get more complicated than in the bulk case considered in Sec. III because we have to evaluate a further two-dimensional integration on the positions $(x, y)$ of the target onions. Details of the calculation can be found in Ref. [27]. 
The total excitation probability $\gamma=\Sigma_{i f} P_{i f}$ was obtained from Eq. (37) by adding the contributions of the transitions from the initial state $i=1 s$ to the excited shells $n_{f}=2$ and 3 for $\mathrm{Li}^{+} @$ and from $i=1 s, 2 s$, and $2 p$ to the final shells $n_{f}=2$ and 3 for $\mathrm{F}^{-} @$. Total values were evaluated by averaging the contributions of different projectile paths. Classical projectile trajectories were derived from Newton's equations corresponding to the punctual potential of Ref. [16] by using the Rung-Kutta method. In the calculation of the projectile-surface interaction, for every position of the projectile we considered contributions from the fourth-order nearest neighboring target ions, which involves four atomic layers of the solid. To avoid the inclusion of new parameters and restrain our calculation to the high energy regime, we overlooked the polarization of the onions by considering only the static potential.

As we are interested in electron emission produced by projectiles that do not penetrate either the bulk or the subsurface region, only trajectories strictly reflected from the topmost atomic plane were selected to evaluate the excitation probability. In all cases 200 projectiles paths with random initial conditions were considered. We found that for incidence directions far away from low-indexed crystallographic channels, as considered here, partial excitation probabilities $P_{i f}$ show a very weak dependence on the particular projectile trajectory.

Figure 7 shows the total number of excitons $\gamma$ produced by grazing protons impinging on a $\mathrm{LiF}$ crystal under random incidence conditions, as a function of the ratio $\theta_{i} / \theta_{c}$, where $\theta_{i}$ is the impact angle measured with respect to the surface plane and $\theta_{c}$ is the critical angle of penetration into the solid. We use $\theta_{c}=0.89$ and $0.34 \mathrm{deg}$ for 100 and $1000 \mathrm{keV}$ impact energies, respectively. We have checked that at these angles $90 \%$ of the trajectories penetrate the first atomic plane. Within the considered angular range, the production of $\mathrm{F}^{-} @$ excitons varies between 7 and 30, while the production of $\mathrm{Li}^{+} @$

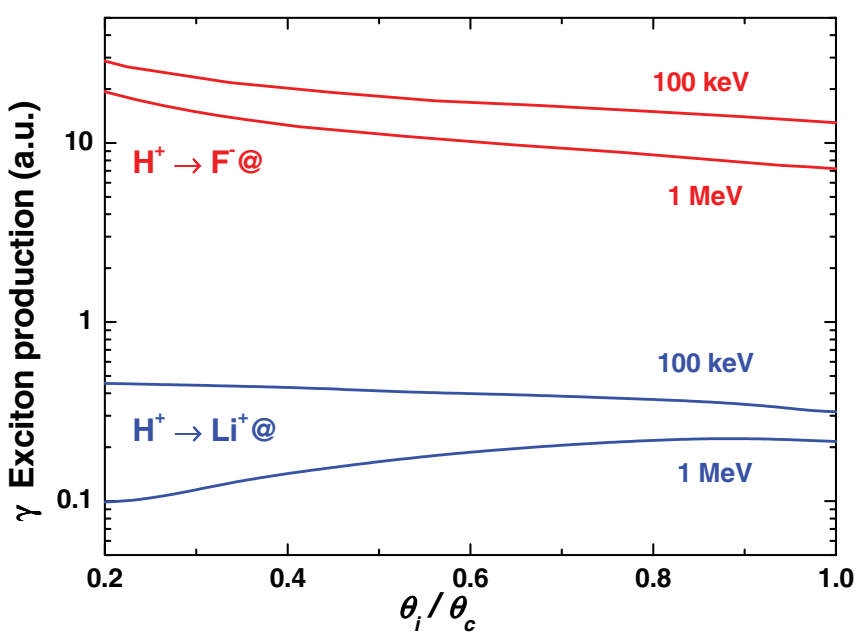

FIG. 7. (Color online) Exciton yields $\gamma$ for grazing proton collisions on $\mathrm{LiF}$ surfaces, as a function of the incident angle $\theta_{i}$ divided by the critical penetration angle $\theta_{c}$, as explained in the text. Two different impact energies are considered: $100 \mathrm{keV}$ and $1 \mathrm{MeV}$. Upper (red) and lower (blue) lines correspond to $\mathrm{F}^{-} @$ and $\mathrm{Li}^{+} @$ targets, respectively. excitons is almost negligible. The small value of the exciton production for $\mathrm{Li}^{+}$@ (the yields are lower than the unity) is due to the fact that grazing projectiles sample regions were only electron densities of $\mathrm{F}^{-} @$ are relevant. In addition, the $\mathrm{F}^{-} @$ states are more loosely bound, producing more excitations, comparatively.

If we consider that the experimental value of the total electron yield is about 60 [34], taking into account that most of the $\mathrm{F}^{-} @$ excitons might decay producing secondary electrons, these values of the exciton production would represent a considerable contribution to the electron emission probability. In practice, we have to consider that the number of excitons could be larger than the primary one calculated here because additional secondary excitons might be produced by primary ejected electrons colliding with $\mathrm{F}^{-} @$ targets. At the bulk it is estimated that one third of the primary ionized electrons end up in excitons and the rest is transformed into atomic vibrations [6]. Therefore, a considerable exchange between exciton and electron production mechanisms is expected.

\section{CONCLUSIONS}

Summing up, we have introduced the concept of the onion potential to simulate the influence of the ionic grid on electrons in a $\mathrm{LiF}$ crystal. Under this assumption it is possible to calculate the binding energies and wave functions of the excited onion states that we have here identified as excitons. Within this framework, excitation and ionization probabilities induced by swift projectiles have been calculated with the help of traditional atomic collision theories, such as the first Born and CDWEIS approximations. The inelastic cross section and the stopping power of protons penetrating the LiF bulk were calculated. The contribution of the exciton production was found to be significant for the inelastic cross section, which is associated with the projectile mean free path, while for the stopping power its contribution was relatively small. From the comparison with stopping experiments we observe a better agreement of the theoretical values derived within the first Born approximation than the ones obtained with the more complex CDWEIS approach. In the case of protons colliding with $\mathrm{LiF}$ surfaces under grazing incidence conditions we found a production between 7 and 30 excitons per incident proton, which might play an important role in electron emission processes.

\section{ACKNOWLEDGMENTS}

The authors would like to acknowledge financial support from CONICET, UBACyT, and ANPCyT of Argentina.

\section{APPENDIX}

As usual, by writing the initial $(i)$ and final $(f)$ unperturbed states as

$$
\varphi_{j}(r)=\frac{u_{j}(r)}{r} Y_{l_{j}}^{m_{j}}(\widehat{r}) \quad \text { with } \quad j=i, f,
$$


the onion form factor given by Eq. (19) can be expressed in terms of a one-dimensional numerical integration as

$$
\begin{aligned}
F_{i f}(\vec{p})= & 4 \pi \sum_{l_{3} m_{3}} i^{l_{3}}(-1)^{m_{f}} D_{l_{f}}^{-m_{f}}{\stackrel{l_{3}}{m_{3}} m_{l_{i}}}_{l_{i}} Y_{l_{3}}^{m_{3}}(\widehat{p}) \\
& \times \int_{0}^{\infty} d r u_{f}(r) \quad j_{l_{3}}(p r) u_{i}(r),
\end{aligned}
$$

where

$$
D_{l_{1}}^{m_{1}} \begin{array}{lll}
m_{2} & m_{3} & m_{3}
\end{array}=\int d \widehat{r} Y_{l_{1}}^{m_{1}}(\widehat{r}) Y_{l_{2}}^{m_{2}}(\widehat{r}) Y_{l_{3}}^{m_{3}}(\widehat{r}) .
$$

In a similar way, the function $\vec{G}_{i f}(\vec{p})$, defined by Eq. (26), is

$$
\begin{aligned}
\vec{G}_{i f}(\vec{p})= & 4 \pi \sum_{l_{3} m_{3}} i^{l_{3}}(-1)^{m_{f}} Y_{l_{3}}^{m_{3}}(\widehat{p})\left[J_{l_{f}}^{-} \vec{V}_{l_{i} l_{3} l_{f}}^{m_{i} m_{3} m_{f}(-)} R_{l_{i} l_{3} l_{f}}^{-}\right. \\
& \left.-J_{l_{f}}^{+} \vec{V}_{l_{i} l_{3} l_{f}}^{m_{i} m_{3} m_{f}(+)} R_{l_{i} l_{3} l_{f}}^{+}\right],
\end{aligned}
$$

with

$$
\begin{gathered}
J_{l}^{-}=\sqrt{\frac{l}{2 l+1}}, \quad J_{l}^{+}=\sqrt{\frac{l+1}{2 l+1}} \\
R_{l_{f} l_{3} l_{i}}^{ \pm}=\int_{0}^{\infty} d r u_{i}(r) j_{l_{3}}(p r) U_{f}^{ \pm}(r), \\
U_{f}^{+}(r)=\frac{d}{d r} u_{f}(r)-\frac{l_{f}+1}{r} u_{f}(r), \\
U_{f}^{-}(r)=\frac{d}{d r} u_{f}(r)+\frac{l_{f}}{r} u_{f}(r) .
\end{gathered}
$$

The coupling vector is

$$
\vec{V}_{l_{i} l_{3} l_{f}}^{m_{i} m_{3} m_{f}( \pm)}=\int d \widehat{r} Y_{l_{i}}^{m_{i}}(\widehat{r}) Y_{l_{3}}^{m_{3}}(\widehat{r}) \vec{Y}_{l_{f 1}, l_{f} \pm 1, m_{f}}(\widehat{r}), \text { (A9) }
$$

where $\vec{Y}_{l_{f 1}, l_{f} \pm 1, m_{f}}(\widehat{r})$ is the spherical harmonic vector as defined in Ref. [35].
[1] P. Roncin, J. Villette, J. P. Atanas, and H. Khemliche, Phys. Rev. Lett. 83, 864 (1999).

[2] H. Khemliche, J. Villette, P. Roncin, and M. Barat, Nucl. Instrum. Methods B 164, 608 (2000).

[3] A. G. Borisov, J. P. Gauyacq, V. Sidis, and A. K. Kazansky, Phys. Rev. B 63, 045407 (2001).

[4] H. Winter, S. Lederer, K. Maass, A. Mertens, F. Aumayr, and H. P. Winter, J. Phys. B 35, 3315 (2002).

[5] H. Winter, A. Mertens, S. Lederer, C. Auth, F. Aumayr, and H. P. Winter, Nucl. Instrum. Methods B 212, 45 (2003).

[6] K. Schwartz, RAU Scientific Reports and Solid State Electronics and Technologies 1, 3-14 (1996) Riga Aviation University, Lomonosov Str. 1, Riga, LV-1019, Latvia.

[7] D. L. Dexter and R. S. Knox, Excitons (John Wiley, New York, 1965).

[8] M. Bader, R. E. Pixley, F. S. Mozer, and W. Whaling, Phys. Rev. 103, 32 (1956). See also M. Bader's thesis, California Institute of Technology, 1955.

[9] M. Draxler, S. P. Chenakin, S. N. Markin, and P. Bauer, Phys. Rev. Lett. 95, 113201 (2005).

[10] E. Clementi and C. Roetti, At. Data Nucl. Data Tables 14, 177 (1974).

[11] M. S. Gravielle and J. E. Miraglia, Phys. Rev. A 71, 032901 (2005).

[12] J. F. Ziegler, J. P. Biersack, and U. Littmark, The Stopping and Range of Ions in Matter (Pergamon, New York, 1985), Vol. 1.

[13] L. Gulyás, P. D. Fainstein, and A. Salin, J. Phys. B 28, 245 (1995).

[14] M. S. Gravielle, I. Aldazabal, A. Arnau, V. H. Ponce, J. E. Miraglia, F. Aumayr, S. Lederer, and H. Winter, Phys. Rev. A 76, 012904 (2007).

[15] C. D. Archubi and M. S. Gravielle, Phys. Rev. A 80, 034901 (2009).

[16] A. J. García and J. E. Miraglia, Phys. Rev. A 75, 042904 (2007).

[17] M. S. Gravielle and J. E. Miraglia, Phys. Rev. A 78, 022901 (2008).
[18] In the GII model, at large distances the active electron of the isolated $\mathrm{F}^{-}$ion see a neutral asymptotic charge, while the electron of $\mathrm{Li}^{+}$ion see an attractive charge +2 .

[19] H. Tatewaki, Surf. Sci. 327, 129 (1995).

[20] G. D. Mahan, Phys. Rev. B 21, 4791 (1980).

[21] F. Salvat, J. M. Fernández-Varea, and W. Williamson Jr., Comput. Phys. Commun. 90, 151 (1995). We like to thank F. Salvat for providing the program RADIALF.

[22] L. Wirtz, M. Dallos, H. Lischka, and J. Burgdorfer, e-print arXiv:cond-mat/0401238 (2004).

[23] H. Tatewaki, Phys. Rev. B 60, 3777 (1999).

[24] Our starting values to derive the potential $V_{\mathrm{F} 0}$, as given by Eq. (10), correspond to the $\mathrm{F}^{-}(2 p)$ state of Clementi and Roetti [10], with a binding energy of $-4.92 \mathrm{eV}$ (see Table III). Instead, the binding energy of the free $\mathrm{F}^{-}(2 p)$ of Ref. [19] is $4.27 \mathrm{eV}$, which makes an starting difference of $0.65 \mathrm{eV}$ between both calculations.

[25] N. W. Ashcroft and N. D. Mermin, Solid State Physics (Thomson Learning, Toronto, 1976).

[26] The $2 s$ and $2 p$ bandwidths of fluoride were estimated as 1.6 and $5.0 \mathrm{eV}$, respectively, while the bandwidth of lithium is negligible.

[27] J. E. Miraglia and M. S. Gravielle, Phys. Rev. A 78, 052705 (2008).

[28] M. S. Gravielle and J. E. Miraglia, Comp. Phys. Commun. 69, 53 (1992).

[29] M. F. Ciappina, W. R. Cravero, and C. R. Garibotti, J. Phys. B 36, 3775 (2003).

[30] R. A. Buckingham and A. Dalgarno, Proc. R. Soc. London Ser. A 213, 506 (1952).

[31] V. M. Kenkre and R. S. Knox, Phys. Rev. Lett. 33, 803 (1974).

[32] W. Hanke and L. J. Sham, Phys. Rev. Lett. 43, 387 (1979).

[33] L. Wirtz, C. Lemell, C. O. Reinhold, L. Häggs, and J. Burgdörfer, Nucl. Instrum. Methods B 182, 36 (2001).

[34] M. S. Gravielle and J. E. Miraglia, Nucl. Instrum. Methods B 258, 21 (2007).

[35] A. R. Edmonds, Angular Momentum in Quantum Mechanics (Princeton University Press, Princeton, NJ, 1974), Eqs. (5.7.2). 